\title{
GEODESIC FLOW IN CERTAIN MANIFOLDS WITHOUT CONJUGATE POINTS
}

\author{
BY \\ PATRICK EBERLEIN( $\left.{ }^{1}\right)$
}

\begin{abstract}
A complete simply connected Riemannian manifold $H$ without conjugate points satisfies the uniform Visibility axiom if the angle subtended at a point $p$ by any geodesic $\gamma$ of $H$ tends uniformly to zero as the distance from $p$ to $\gamma$ tends uniformly to infinity. A complete manifold $M$ is a uniform Visibility manifold if it has no conjugate points and if the simply connected covering $H$ satisfies the uniform Visibility axiom. We derive criteria for the existence of uniform Visibility manifolds. Let $M$ be a uniform Visibility manifold, $S M$ the unit tangent bundle of $M$ and $T_{t}$ the geodesic flow on $S M$. We prove that if every point of $S M$ is nonwandering with respect to $T_{t}$ then $T_{t}$ is topologically transitive on $S M$. We also prove that if $M^{\prime}$ is a normal covering of $M$ then $T_{t}$ is topologically transitive on $S M^{\prime}$ if $T_{t}$ is topologically transitive on $S M$.
\end{abstract}

Introduction. Much research, past and present, has been devoted to proving the topological transitivity of the geodesic flow in manifolds with curvature $K \leqq 0$ that satisfy various conditions, usually including compactness. An essential part of all the proofs is the fact that if $M$ is a complete manifold with $K \leqq 0$ and simply connected covering space $H$ then any two distinct points of $H$ are joined by a unique geodesic. Manifolds $M$ without conjugate points are characterized by this property in the simply connected covering space $H$ and it is natural to ask what further conditions on $M$ are sufficient to imply that the geodesic flow is topologically transitive. Results have been obtained (mostly in the compact case) by Green, Hedlund, Klingenberg, Morse and others under the assumption that the manifold $M$ admits another (closely related) metric $g^{*}$ with curvature $K \equiv-1$ or $K \leqq c<0$.

In this paper we extend and unify many of these results by requiring that $M$ be a uniform Visibility manifold (defined above and more precisely in $\$ 1$ ). To complement the main results stated above we obtain the following criteria for the existence of uniform Visibility manifolds.

THEOREM. Let $M$ be a compact manifold with curvature $K \leqq 0$. Then $M$ is a uniform Visibility manifold if and only if the simply connected covering $H$ contains no totally geodesic isometric imbedding of the plane $R^{2}$.

Received by the editors March 23, 1971.

AMS 1970 subject classifications. Primary 53A35, 53C20; Secondary 28A65, 34C35, SPF 99.

Key words and phrases. Geodesic flow, conjugate points, nonwandering points, topological transitivity, uniform Visibility.

( $\left.{ }^{1}\right)$ This research was supported in part by NSF Grants GP-11476 and GP-20096.

Copyright (C) 1972, American Mathematical Society 
THEOREM $\left({ }^{2}\right)$. Let $M$ be a compact uniform Visibility manifold with respect to a metric $g$. If $g^{*}$ is any other metric on $M$ that admits no conjugate points, then $M$ is a uniform Visibility manifold with respect to the metric $g^{*}$.

The theorems above imply that a uniform Visibility manifold may admit some positive sectional curvature. For example, if $(M, g)$ is compact with sectional curvature $K<0$, then $(M, g)$ is a uniform Visibility manifold. If $g^{*}$ is any metric without conjugate points. on $M$ then $\left(M, g^{*}\right)$ is a uniform Visibility manifold and may have some positive sectional curvature $\left({ }^{3}\right)$. To explicitly construct metrics $g^{*}$ with some positive curvature one might alter the original metric $g$ in a small neighborhood on $M$, for example by conformally changing $g$ by a $C^{\infty}$ function $f: M \rightarrow R$ that is the identity outside a small neighborhood on $M$. Hopefully if the neighborhood is sufficiently small and if the function $f$ is chosen suitably, then $g^{*}$ will have no conjugate points and $M$ will have some positive curvature within the neighborhood. The simplest candidates for this technique are the compact surfaces of genus $n \geqq 2$. Each such surface $M$ admits a metric $g$ with curvature $K \equiv-1$.

Proofs of the theorems above may be found in $\$ \S 4$ and 5. The first and third theorems extend results of Anosov in one direction. The first, third and fourth theorems extend many of the classical results referred to above. The second theorem provides examples of manifolds of infinite volume for which the geodesic flow is topologically transitive.

The basic task of this paper is to extend the machinery of points at infinity developed in [3] for manifolds with curvature $K \leqq 0$ to uniform Visibility manifolds. The proofs of [3] must be modified since we no longer have the convexity properties of negative curvature, but the uniform Visibility axiom is a sufficient replacement. We represent $M$ as a quotient manifold $H / D$ where $H$ is simply connected and $D$ is a properly discontinuous group of isometries of $H$. As in [3] we define $H(\infty)$, the points at infinity for $H . \bar{H}=H \cup H(\infty)$ is topologically an $n$-cell. We define the limit set of $D, L(D)$, a closed $D$-invariant subset of $H(\infty)$. One may show as in [3] that $L(D)$ is precisely the set of points in $\bar{H}$ where $D$ fails to act properly discontinuously. After investigating other of its properties we relate $L(D)$ to the geodesic flow in the unit tangent bundle of $M=H / D$.

1. Points at infinity. Let $M$ be a complete Riemannian manifold and let $\sigma: I \rightarrow M$ be a $C^{\infty}$ curve. A vector field $Y$ on $\sigma$ is a Jacobi vector field if

$$
Y^{\prime \prime}+R_{X Y} X=0
$$

where $X$ is the tangent vector field of $\sigma, R$ is the curvature tensor of $M$ and ' denotes covariant differentiation with respect to $X$. Points $p$ and $q$ on $\sigma$ are conjugate along $\sigma$ if there exists a nonzero Jacobi vector field on $\sigma$ that vanishes at $p$ and $q$.

$\left({ }^{2}\right)$ See Added in proof.

( ${ }^{3}$ See Added in proof. 
$M$ is a manifold without conjugate points if for any geodesic $\sigma$ of $M$ no two points of $\sigma$ are conjugate along $\sigma$. It is well known that if $M$ has no conjugate points then, for each point $p$ in $M$, $\exp _{p}: M_{p} \rightarrow M$ is a covering map. If $M$ is simply connected then $\exp _{p}$ is a diffeomorphism and any two distinct points of $M$ are joined by a unique geodesic. In the sequel, $H$ will denote a complete, simply connected manifold without conjugate points, $M$ an arbitrary complete manifold without conjugate points. All geodesics in $M$ or $H$ are assumed to have unit speed.

If $M$ is not simply connected $M$ can be represented as a quotient manifold $H / D$ where $D$ is a properly discontinuous group of isometries of $H$, isomorphic in a natural way to the fundamental group of $M$. We shall study the geodesics of $M$ by investigating the action of $D$ on $H$. We now define some useful concepts.

Definition 1.1. If $p$ and $q$ are distinct points of $H$ then $V(p, q)$ is the unique unit vector $v$ at $p$ such that $q=\exp _{p}(t v)$ where $t=d(p, q) . d$ is the Riemannian distance function of $M$.

Definition 1.2. If $q, r$ are points of $H$ distinct from a point $p$ then $\chi_{p}(q, r)$ is the angle subtended by $V(p, q)$ and $V(p, r)$, the value being taken between 0 and $\pi$.

Definition 1.3. $H$ satisfies the Visibility axiom if for each point $p \in H$ and $\varepsilon>0$ there exists a constant $R=R(p, \varepsilon)>0$ such that if $\sigma:[a, b] \rightarrow H$ is a geodesic segment satisfying the condition $d(p, \sigma) \geqq R$ then $\chi_{p}(\sigma a, \sigma b) \leqq \varepsilon . H$ satisfies the uniform Visibility axiom if the constant $R$ may be chosen to be independent of $p$. $M$ is said to satisfy the (uniform) Visibility axiom if its simply connected Riemannian cover $H$ does. Such an $M$ is a (uniform) Visibility manifold.

An examination of the proof of Proposition 5.9 in [3] shows that the uniform Visibility axiom is satisfied by complete manifolds with $K \leqq c<0$, in particular by hyperbolic space. The Visibility axiom alone appears to be a sufficient replacement in manifolds with $K \leqq 0$ for the curvature condition $K \leqq c<0$ in many classical results. It is also shown in [3] that if $H$ has $K \leqq 0$ and satisfies the Visibility axiom and if $A \subseteq H$ is any compact subset then we can choose $R$ depending only on $A$ and $\varepsilon$; briefly the Visibility axiom is uniform on compact subsets. If $\phi$ is an isometry of $H$ then we may choose $R(\phi p, \varepsilon)=R(p, \varepsilon)$. Hence for compact manifolds with $K \leqq 0$ the Visibility and uniform Visibility axioms are equivalent. Very likely this is true for compact manifolds without conjugate points but at present we cannot prove this.

Henceforth we assume that $H$ satisfies the uniform Visibility axiom.

By adding a boundary sphere $H(\infty)$ we compactify $H$ as was done in [3] for manifolds of curvature $K \leqq 0$. The proofs, though similar to those of [3], must be modified since the law of cosines is not true in general. The uniform Visibility axiom is a sufficient replacement and the geometric conditions it imposes also yield strong results about the geodesics of $M=H / D$.

Definition 1.4. Geodesics $\gamma, \sigma$ in $H$ are asymptotes if there exists a constant $c>0$ such that $d(\gamma t, \sigma t) \leqq c$ for $t \geqq 0$. 
Clearly the asymptote relation is an equivalence relation on the geodesics of $H$. Let $\gamma(\infty)$ denote the equivalence class of the geodesic $\gamma$. Let $\gamma(-\infty)$ denote the equivalence class of $\gamma^{-1}: \gamma^{-1}(t)=\gamma(-t)$. A point at infinity for $H$ is an equivalence class of geodesics of $H$ and $H(\infty)$ is the set of all points at infinity. A geodesic $\gamma$ is said to join points $x$ and $y$ in $H(\infty)$ if $\gamma(\infty)=y$ and $\gamma(-\infty)=x$. Let $\bar{H}=H \cup H(\infty)$.

Proposition 1.5. Let $\gamma$ be a geodesic in $H$ and $p$ any point of $H$. Then there exists a unique geodesic $\sigma$ such that $\sigma(0)=p$ and $\sigma$ is asymptotic to $\gamma$.

Proof. We show uniqueness first. If $\sigma_{1}, \sigma_{2}$ are geodesics that start at $p$ and are asymptotic to $\gamma$ then there exists $c>0$ such that $d\left(\sigma_{1} t, \sigma_{2} t\right) \leqq c$ for $t \geqq 0$. By the uniform Visibility axiom $\chi_{p}\left(\sigma_{1} t, \sigma_{2} t\right) \rightarrow 0$ as $t \rightarrow \infty$. Since $V\left(p, \sigma_{1} t\right)$ and $V\left(p, \sigma_{2} t\right)$ are constant in $t, \sigma_{1}=\sigma_{2}$. The existence of such asymptotes follows from

LEMMA 1.6. Let $p_{n}, q_{n}, r_{n}$ be sequences in $H$ such that $q_{n} \rightarrow q, r_{n} \rightarrow r$ and $p_{n}$ is divergent. Let $\gamma, \sigma$ be geodesics with initial velocities $v, w$ such that $V\left(q_{n}, p_{n}\right) \rightarrow v$ and $V\left(r_{n}, p_{n}\right) \rightarrow w$. Then $\gamma$ and $\sigma$ are asymptotes.

Proof. Let $A$ be a constant such that if $\sigma$ is a geodesic segment whose endpoints subtend an angle $\geqq \pi / 2$ at a point $p$ then $d(p, \sigma) \leqq A$. Let $\gamma_{n}$ be the geodesic with initial velocity $V\left(q_{n}, p_{n}\right)$. Since $d\left(q_{n}, r_{n}\right)$ is bounded the uniform Visibility axiom implies that there exists a constant $T>0$ such that, for $t \geqq T$ and every integer $n$, $\chi_{\gamma_{n} t}\left(q_{n}, r_{n}\right) \leqq \pi / 2$. Fix $t \geqq T$. For any integer $n>0$ and $s>t$ let $\sigma_{n}^{s}$ denote the geodesic with initial velocity $V\left(r_{n}, \gamma_{n} s\right)$. Since $\chi_{\gamma_{n} t}\left(\gamma_{n} s, r_{n}\right) \geqq \pi / 2, d\left(\gamma_{n} t, \sigma_{n}^{s}\right) \leqq A$. As $s \rightarrow \infty$ any geodesic $\sigma_{n}$ that is a limit point of the geodesics $\sigma_{n}^{s}$ satisfies $d\left(\gamma_{n} t, \sigma_{n}\right) \leqq A$ by continuity. Since $t \geqq T$ was arbitrary it follows from the definition of $\sigma_{n}$ that $d\left(\gamma_{n} t, \sigma_{n}\right) \leqq A$ for every $t \geqq T$ and every integer $n$. Since $d\left(q_{n}, r_{n}\right)$ is bounded we may choose $C>0$ such that for $t \geqq 0$ and every integer $n, d\left(\gamma_{n} t, \sigma_{n}\right) \leqq C$. Since $p_{n}=\gamma_{n}\left(t_{n}\right)$ where $t_{n} \rightarrow \infty$, uniform Visibility implies that the angle subtended by $\sigma_{n}^{\prime}(0)$ and $V\left(r_{n}, p_{n}\right) \rightarrow 0$. Hence $\sigma_{n}^{\prime}(0) \rightarrow w=\sigma^{\prime}(0)$ and it follows that $d(\gamma t, \sigma) \leqq C$ for $t \geqq 0$. The triangle inequality now shows that $d(\gamma t, \sigma t)$ is bounded above for $t \geqq 0$.

Proposition 1.7. If $x, y$ are distinct points in $H(\infty)$ then there exists a geodesic $\sigma: \sigma(-\infty)=x$ and $\sigma(\infty)=y$.

Proof. Fix $p \in H$ and let $\gamma, \rho$ be the unique geodesics such that $\gamma(0)=\rho(0)=p$, $\gamma(\infty)=x$ and $\rho(\infty)=y$. Let $\alpha_{n}$ be the unique geodesic segment from $\gamma(n)$ to $\rho(n)$ for every integer $n>0$. Let $q_{n}$ be a point on $\alpha_{n}$ closest to $p$ and parametrize $\alpha_{n}$ so that $\alpha_{n}(0)=q_{n}$. By uniform Visibility $d\left(p, q_{n}\right)$ is bounded hence $\alpha_{n}^{\prime}(0) \rightarrow v$, passing to a subsequence if necessary. If $\sigma$ is the geodesic with $\sigma^{\prime}(0)=v$ then $\sigma(-\infty)=x$ and $\sigma(\infty)=y$ by Lemma 1.6 .

We now extend the domain of the vector field $V$ of Definition 1.1 and define an angular measure for points at infinity.

Definition 1.8. If $p \in H$ and $x \in H(\infty)$ then $V(p, x)$ is the initial velocity of the unique geodesic $\sigma$ such that $\sigma(0)=p$ and $\sigma(\infty)=x$. 
Definition 1.9. Let $p \in H$ and let $q, r$ be points of $\bar{H}=H \cup H(\infty)$ that are distinct from $p$. Then $\chi_{p}(q, r)$ is the angle subtended by $V(p, q)$ and $V(p, r)$.

An easy consequence of Lemma 1.6 is the following:

LEMMA 1.10. Let $p \in H, x \in H(\infty)$ be given. If $\sigma$ is a geodesic of $H$ such that $\sigma(\infty)=x$ then $V(p, \sigma t) \rightarrow V(p, x)$ as $t \rightarrow \infty$.

Corollary 1.11. Let $p \in H, x, y \in H(\infty)$ be given. If $\sigma$ is a geodesic of $H$ such that $\sigma(\infty)=x$ then $\chi_{p}(\sigma t, y) \rightarrow \chi_{p}(x, y)$ as $t \rightarrow \infty$.

We now define a natural topology on $\bar{H}$. Let $p \in H, v$ a unit vector at $p, 0<\varepsilon<\pi$ and $R>0$ be given. The truncated cone $T$ with vertex $p$, axis $v$, angle $\varepsilon$ and radius $R$ is the set $\{q \in \bar{H}: d(p, q)>R$ and the angle subtended by $v$ and $V(p, q)$ is $<\varepsilon\}$. The distance condition is vacuous for points in $H(\infty)$.

PROPOSITION 1.12.

(1) The open sets of $H$ and the truncated cones form a basis for a topology of $\bar{H}$.

(2) For any point $p \in H$ the truncated cones with vertex $p$ form a local basis for this topology at points of $H(\infty)$.

Proof. Since the intersection of two truncated cones with vertex $p$ contains a truncated cone with vertex $p$, it suffices to prove that for any point $p \in H$, any truncated cone $T$ and any $x \in T \cap H(\infty)$ there exists a truncated cone $S$ with vertex $p$ such that $x \in S \subseteq T$. Let $T$ have radius $R$ and vertex $r$. For some $\delta>0, B=\{q \in \bar{H}$ : $d(q, r)>R$ and $\left.\chi_{r}(q, x) \leqq \delta\right\}$ is contained in $T$. We show first that there exists a truncated cone $S$ with vertex $p$ such that $x \in S$ and $S \cap H \subseteq B$. If this were false then there would exist a sequence $q_{n} \subseteq H$ such that $\chi_{p}\left(q_{n}, x\right) \rightarrow 0, d\left(p, q_{n}\right) \rightarrow \infty$ and $q_{n} \notin B$ for any integer $n$. Passing to a subsequence $V\left(r, q_{n}\right) \rightarrow v \neq V(r, x)$. Since $V\left(p, q_{n}\right) \rightarrow V(p, x)$, Lemma 1.6 implies that the geodesics with initial velocities $v$ and $V(p, x)$ are asymptotes, but this contradicts the definition of $V(r, x)$. Hence for some truncated cone $S$ with vertex $p$ and radius $A, x \in S$ and $S \cap H \subseteq B$. Let $y \in S \cap H(\infty)$ and let $\sigma$ be the unique geodesic such that $\sigma(0)=p$ and $\sigma(\infty)=y$. For $t>A, \sigma(t) \in S \cap H \subseteq B$ and $\chi_{r}(x, \sigma t) \leqq \delta$. By Corollary 1.11, $\chi_{r}(x, \sigma t) \rightarrow \chi_{r}(x, y)$ $\leqq \delta$ as $t \rightarrow \infty$. Hence $y \in B$ and $S \subseteq B \subseteq T$.

In accordance with [3] we call this topology the cone topology for $\bar{H}$.

Proposition 1.13. $\bar{H}$ is homeomorphic to the closed unit $n$-ball

$$
B=\left\{x \in R^{n}:\|x\| \leqq 1\right\} .
$$

Proof. Fix $p \in H$ and let $B_{p}$ be the set of tangent vectors at $p$ of norm $\leqq 1$.

Define $F: B_{p} \rightarrow \bar{H}$ by

$$
\begin{array}{ll}
F(v)=\exp _{p}(v /(1-\|v\|)) & \text { if }\|v\|<1, \\
F(v)=\gamma_{v}(\infty) & \text { if }\|v\|=1,
\end{array}
$$

where $\gamma_{v}$ denotes the geodesic with initial velocity $v$. The continuity of $F$ is immediate since the truncated cones at $p$ are a local basis at $H(\infty)$. It is easy to see that $F$ is a bijection. Since $B_{p}$ is compact and $\bar{H}$ is Hausdorff, $F$ is a homeomorphism. 
Proposition 1.14. Let $A=\{(p, q) \in H \times \bar{H}: p \neq q\}$. Then the function $(p, q)$ $\rightarrow V(p, q)$ is continuous.

Proof. If $(p, q) \in H \times H$ continuity follows from the continuity of the exponential map. Suppose that $(p, q) \in H \times H(\infty)$. It suffices to show that if $\left(p_{n}, q_{n}\right) \subseteq A$ converges to $(p, q)$ then $V\left(p_{n}, q_{n}\right)$ converges to $V(p, q)$. Let $q_{n}^{\prime} \subseteq H$ be chosen so that $d\left(p_{n}, q_{n}^{\prime}\right) \rightarrow \infty$ and $V\left(p_{n}, q_{n}^{\prime}\right)=V\left(p_{n}, q_{n}\right)$. Hence we may assume without loss of generality that $q_{n} \subseteq H$. Let $v$ be a cluster point of $V\left(p_{n}, q_{n}\right)$, the limit of a subsequence $V\left(p_{n_{k}}, q_{n_{k}}\right)$. Passing to a further subsequence let $V\left(p, q_{n_{k}}\right)$ converge to $v^{*}$. By Lemma 1.6, $v=v^{*}$. However $V\left(p, q_{n_{k}}\right) \rightarrow V(p, q)$ since $q_{n_{k}} \rightarrow q$. Hence $V(p, q)$ is the only cluster point of $V\left(p_{n}, q_{n}\right)$.

Proposition 1.15. Let $v_{n}$ be a sequence of unit vectors in $H$ that converge to a vector $v$. Let $t_{n} \rightarrow+\infty$. Then $\gamma_{v_{n}}\left(t_{n}\right) \rightarrow \gamma_{v}(\infty)$.

Proof. Let $q_{n}=\gamma_{v_{n}}\left(t_{n}\right), p_{n}=$ the point of tangency of $v_{n}, p=$ the point of tangency of $v$ and $x=\gamma_{v}(\infty)$. Then $V\left(p_{n}, q_{n}\right)=v_{n} \rightarrow v=V(p, x)$. It follows that $q_{n} \rightarrow x$ by the continuity of $V$.

2. Limit sets. Let $D$ denote a properly discontinuous group of isometries of $H$. We extend the action of $D$ to $\bar{H}$ and describe its behavior on $H(\infty)$.

If $\phi$ is an isometry of $H$ we extend $\phi$ to act on $\bar{H}$ by requiring that $\phi\{\gamma(\infty)\}$ $=(\phi \circ \gamma)(\infty)$ for any geodesic $\gamma . \phi$ is well defined since isometries preserve distance. $\phi$ is a homeomorphism since $\phi$ carries truncated cones into truncated cones. Since $\bar{H}$ is an $n$-cell every isometry $\phi$ of $H$ without fixed points in $H$ has a fixed point in $H(\infty)$. If $D$ is a properly discontinuous group of isometries of $H$ then $D$ extends to a group of homeomorphisms of $\bar{H}$. D is no longer properly discontinuous on $\bar{H}$ and the points where proper discontinuity fails are especially interesting.

Definition 2.1. For a group $D$ let $L(D)$ be the set of accumulation points in $\bar{H}$ of an orbit $D(p)$ where $p$ is a point of $H . L(D)$ is called the limit set of $D$.

By the uniform Visibility axiom $L(D)$ does not depend on the particular point $p$. $L(D)$ is a closed set in $H(\infty)$ that is invariant under $D$. One may show as in [3] that $L(D)$ is precisely the set of points in $\bar{H}$ where proper discontinuity fails, but this fact will not be needed. The following result is crucial to the investigation of the action of $D$ on $H(\infty)$. The proof may also be found in [3].

If $p \notin A \subseteq \bar{H}$ the angle $\chi_{p}(A)$ subtended by $A$ at $p=\sup \left\{\chi_{p}(a, b): a, b \in A\right\}$.

Proposition 2.2. Let $x \in H(\infty)$ and let $U$ be an open set of $\bar{H}$ containing $x$. If $p_{n} \subseteq H$ is a sequence such that $p_{n} \rightarrow x$ then $\chi_{p_{n}}(\bar{H}-U) \rightarrow 0$.

Proof. It suffices to prove that for any sequences $a_{n}, b_{n}$ in $\bar{H}-U, \chi_{p_{n}}\left(a_{n}, b_{n}\right)$ $\rightarrow 0$. Let $p \in H-U$. It suffices to show that $\chi_{p_{n}}\left(a_{n}, p\right) \rightarrow 0$ for any sequence $a_{n}$ in $\bar{H}-U$. By Proposition 1.12 we may assume that $U$ is a truncated cone with vertex $p$. Let $\sigma_{n}$ be the unique geodesic from $p_{n}$ to $a_{n}$. Since $a_{n} \in \bar{H}-U$ there exists $\varepsilon>0$ such that, for sufficiently large $n, \chi_{p}\left(p_{n}, a_{n}\right) \geqq \varepsilon>0$. By uniform Visibility there exists $R>0$ such that $d\left(p, \sigma_{n}\right) \leqq R$ for every integer $n$. Let $q_{n}$ be a point 
on $\sigma_{n}$ such that $d\left(p, q_{n}\right) \leqq R$. For large $n, q_{n} \neq p_{n}$ and $\chi_{p_{n}}\left(q_{n}, p\right)=\chi_{p_{n}}\left(a_{n}, p\right)$. But by uniform Visibility $\chi_{p_{n}}\left(q_{n}, p\right) \rightarrow 0$ since $d\left(p, q_{n}\right)$ is bounded.

The following concept will be related to properties of the geodesic flow in the unit tangent bundle of $M=H / D$.

Definition 2.3. Points $x$ and $y$ in $H(\infty)$, not necessarily distinct, are dual with respect to $D$ if for any open sets $U, V$ containing $x, y$ respectively there exists an element $\phi \in D$ such that $\phi(\bar{H}-U) \subseteq V$.

Clearly $\phi(\bar{H}-U) \subseteq V$ if and only if $\phi^{-1}(\bar{H}-V) \subseteq U$. The following result is obvious.

Proposition 2.4. Let $x \in H(\infty)$. The set of points dual to $x$ is closed in $H(\infty)$ and invariant under $D$.

A consequence of the following proposition is that dual points lie in $L(D)$ and that any point in $L(D)$ has a dual point.

Proposition 2.5. Points $x, y$ in $H(\infty)$ are dual if and only if there exists a sequence $\phi_{n} \subseteq D$ such that, for any point $p \in H, \phi_{n}^{-1}(p) \rightarrow x$ and $\phi_{n}(p) \rightarrow y$.

Proof. Suppose that $x$ and $y$ are dual and let $\left\{U_{n}\right\},\left\{V_{n}\right\}$ be local bases for the cone topology at $x$ and $y$ respectively. For each integer $n$ there exists $\phi_{n} \in D$ such that $\phi_{n}\left(\bar{H}-U_{n}\right) \subseteq V_{n}$ and $\phi_{n}^{-1}\left(\bar{H}-V_{n}\right) \subseteq U_{n}$. For any point $p \in H, p \in\left(\bar{H}-U_{n}\right)$ $\cap\left(\bar{H}-V_{n}\right)$ for sufficiently large $n$ and hence $\phi_{n}(p) \in V_{n}$ and $\phi_{n}^{-1}(p) \in U_{n}$. Thus $\phi_{n}(p) \rightarrow y$ and $\phi_{n}^{-1}(p) \rightarrow x$. Conversely suppose that such a sequence $\phi_{n} \subseteq D$ exists. Let $U, V$ be open sets containing $x$ and $y$ respectively. By Proposition 2.2, $\chi_{p} \phi_{n}(\bar{H}-U)=\chi_{\phi_{n}^{-1}} p(\bar{H}-U) \rightarrow 0$ for any point $p \in H$. We may assume that $p \in \bar{H}-U$. Since $\phi_{n}(p) \rightarrow y$ and $d\left(\phi_{n}^{-1} p, \bar{H}-U\right) \rightarrow \infty$ it follows that $\phi_{n}(H-U) \subseteq V$ for sufficiently large $n$. Thus $x$ and $y$ are dual since $U, V$ were arbitrary.

Our goal is to prove that if $L(D)$ has at least three points then any two points of $L(D)$ (not necessarily distinct) are dual. We first investigate the fixed point sets of elements of $D$. If $\phi \in D$ and $p \in H$ are given then any cluster point of the sequence $\left\{\phi^{n}(p): n\right.$ is an integer $\}$ is a fixed point of $\phi$. To show this let $\phi^{n_{k}}(p) \rightarrow x$. Then $\phi^{1+n_{k}}(p) \rightarrow \phi x$. Since $d\left(\phi^{n_{k}} p, \phi^{\left.1+n_{k} p\right)}=d(p, \phi p)\right.$ it follows that $\chi_{p}\left(\phi^{n_{k}} p, \phi^{1+n_{k} p}\right)$ $\rightarrow 0$ by uniform Visibility. Thus $\phi x=x$.

The following proposition shows that $\phi$ has at most two fixed points and these are of the kind above. Hence all fixed points of elements of $D$ lie in $L(D)$.

Proposition 2.6. Let $D$ be a properly discontinuous group of isometries of $H$. Let $\phi, \phi^{*}$ be elements of $D$.

(1) If $\phi$ has fixed points $x, y$ in $H(\infty)$ then (replacing $\phi$ by $\phi^{-1}$ if necessary) $\phi^{n}(q) \rightarrow x$ and $\phi^{-n}(q) \rightarrow y$ for any point $q \in H$.

(2) $\phi$ has at most two fixed points in $H(\infty)$.

(3) Suppose that $\phi, \phi^{*}$ have fixed point sets $\{x, y\}$ and $\left\{x^{*}, y^{*}\right\}$ respectively in $H(\infty)$. Then either these sets are disjoint or they are identical. 
Proof. (1) By Proposition 1.7 there exists a geodesic $\gamma$ such that $\gamma(-\infty)=y$ and $\gamma(\infty)=x$. For a point $p$ on $\gamma$ and any integer $n$

$$
\chi_{\phi^{n} p}(x, y)=\chi_{p}\left(\phi^{-n} x, \phi^{-n} y\right)=\chi_{p}(x, y)=\pi .
$$

Hence there exists $R>0$ such that $d\left(\phi^{n} p, \gamma\right) \leqq R$ for every integer $n$ by uniform Visibility. Choose sequences of numbers $s_{n}, t_{n}$ such that $d\left(\phi^{n} p, \gamma s_{n}\right) \leqq R$ and $d\left(\phi^{-n} p, \gamma t_{n}\right) \leqq R$ for all integers $n \geqq 0$. By proper discontinuity $s_{n}$ and $t_{n}$ have no finite accumulation points. Suppose that some subsequence $s_{n_{k}} \rightarrow \infty$. We show that $\phi^{n} p \rightarrow x$ and $\phi^{-n} p \rightarrow y$. For any $t \geqq 0$ there is a smallest $n>0$ such that $t<s_{n}$ hence $\bigcup_{n \geqq 0}\left[s_{n}, s_{n+1}\right]$ covers $[0, \infty)$ where $s_{0}=0$.

For any $n$,

$$
\begin{aligned}
\left|s_{n}-s_{n+1}\right| & =d\left(\gamma s_{n}, \gamma s_{n+1}\right) \leqq d\left(\gamma s_{n}, \phi^{n} p\right)+d\left(\phi^{n} p, \phi^{n+1} p\right)+d\left(\phi^{n+1} p, \gamma s_{n+1}\right) \\
& \leqq 2 R+d(p, \phi p)=C .
\end{aligned}
$$

By proper discontinuity we may choose $N>0$ such that $n \geqq N$ implies $d\left(p, \phi^{n} p\right)$ $\geqq 2 C+2 R$. Then for $k \geqq N$ and any $n \geqq 0,\left|t_{k}-s_{n}\right| \geqq d\left(\phi^{-k} p, \phi^{n} p\right)-2 R \geqq 2 C$. Hence $t_{k}<0$ for $k \geqq N$ and $t_{k} \rightarrow-\infty$. Reversing the argument we show that $s_{n} \rightarrow+\infty$. By uniform Visibility $\chi_{p}\left(\phi^{n} p, \gamma s_{n}\right) \rightarrow 0$ and $\chi_{p}\left(\phi^{-n} p, \gamma t_{n}\right) \rightarrow 0$. Hence $\phi^{n} p \rightarrow x$ and $\phi^{-n} p \rightarrow y$ since $\gamma(t) \rightarrow \gamma(\infty)$ as $t \rightarrow \infty$ and $\gamma(-t) \rightarrow \gamma(-\infty)$ as $t \rightarrow \infty$. By uniform Visibility $\phi^{n} q \rightarrow x$ and $\phi^{-n} q \rightarrow y$ for any $q \in H$.

(2) The proof follows directly from (1).

(3) Suppose that $y=y^{*}$. Replacing $\phi$ or $\phi^{*}$ by their inverses if necessary, it follows from (1) that, for any $p \in H, \phi^{n} p \rightarrow y$ and $\phi^{* n} p \rightarrow y$. Let $\gamma, \gamma^{*}$ be geodesics joining $x$ to $y$ and $x^{*}$ to $y$ respectively. Fix a point $p \in H$. As in the proof of (1) we may choose $C>0$ and sequences $s_{n}, t_{n}$ diverging to $+\infty$ such that $d\left(\phi^{n} p, \gamma s_{n}\right) \leqq C$, $d\left(\phi^{* n} p, \gamma^{*} t_{n}\right) \leqq C,\left|s_{n}-s_{n+1}\right| \leqq C$ and $\bigcup_{n \geqq 0}\left[s_{n}, s_{n+1}\right]$ covers $[0, \infty)$.

For each integer $k>0$ there exists $s_{n_{k}}$ such that $\left|s_{n_{k}}-t_{k}\right| \leqq C$. Since $\gamma$ and $\gamma^{*}$ are asymptotes there exists $R>0$ such that, for $t \geqq 0, d\left(\gamma t, \gamma^{*} t\right) \leqq R$. For each $k>0$,

$$
\begin{aligned}
d\left(p, \phi^{\left.-n_{k} \phi^{* k} p\right)}\right. & =d\left(\phi^{n_{k}} p, \phi^{* k} p\right) \\
& \leqq d\left(\phi^{n_{k}} p, \gamma s_{n_{k}}\right)+d\left(\gamma s_{n_{k}}, \gamma t_{k}\right)+d\left(\gamma t_{k}, \gamma^{*} t_{k}\right)+d\left(\gamma^{*} t_{k}, \phi^{* k} p\right) \leqq 3 C+R .
\end{aligned}
$$

By proper discontinuity only finitely many of the elements $\left\{\phi^{-n_{k}} \phi^{* k}\right\}$ are distinct, hence $\phi^{r}=\phi^{* s}$ for suitable integers $r$ and $s . \phi^{r}$ fixes $x, y$ and $x^{*}$ which implies that $x=x^{*}$ by (2) and the fact that $x^{*} \neq y^{*}=y$.

Proposition 2.7. Let $x, y$ be distinct dual points in $H(\infty)$. Let $U, V$ be neighborhoods of $x, y$ respectively. Then there exists an element $\phi \in D$ such that $\phi$ has fixed points in $U \cap H(\infty)$ and $V \cap H(\infty)$.

Proof. We may assume that $U, V$ are truncated cones with a common vertex $p$ and that $\bar{U}$ and $\bar{V}$ are disjoint. Using the homeomorphism of Proposition 1.13 we see that $\bar{U} \cap H(\infty)$ and $\bar{V} \cap H(\infty)$ are homeomorphic to convex neighborhoods on $S^{n-1}$ and hence to closed $(n-1)$-disks. By duality there exists an element $\phi$ of $D$ 
such that $\phi(\bar{H}-V) \subseteq U$. Hence $\phi(\bar{U}) \subseteq U$ and $\phi(\bar{U} \cap H(\infty)) \subseteq U \cap H(\infty)$. By the Brouwer fixed point theorem $\phi$ has a fixed point in $U \cap H(\infty)$. Similarly $\phi^{-1}(\bar{V})$ $\subseteq V$ and $\phi^{-1}$ (hence $\phi$ ) has a fixed point in $V \cap H(\infty)$.

We now reach the main result of this section. We note that $L(D)$ is actually an infinite set if it contains three points.

Proposition 2.8. If $L(D)$ contains at least three points then any two points of $L(D)$ are dual.

Proof. We first show that there exists some point $z^{*} \in L(D)$ such that $z^{*}$ is dual to every point of $L(D)$. Since $L(D)$ has at least three points we can choose some $z \in L(D)$ that is not a common fixed point of $D$. We show that $D(z)$ is dense in $L(D)$. Let $y \in L(D)$ be given and let $U$ be a neighborhood of $y$. By the remark preceding Proposition $2.5, y$ has a dual point $y^{*}$. Since $D(z)$ contains at least two points we may choose a neighborhood $V$ of $y^{*}$ such that $\phi(z) \in \bar{H}-V$ for some $\phi \in D$. Choose $\psi \in D$ such that $\psi(\bar{H}-V) \subseteq U$. Then $\psi \phi(z) \in U$ and this proves that $D(z)$ is dense in $L(D)$. If $z^{*}$ is dual to $z$ then, by Proposition $2.4, z^{*}$ is dual to every point in $L(D)$. Let $x \neq z^{*}$ be given in $L(D)$. We show that $z^{*}$ is not a common fixed point of $D$. It will then follow as above that $D\left(z^{*}\right)$ is dense in $L(D)$ and that $x$ is dual to every point of $L(D)$ since $x$ is dual to $z^{*}$. Choose $y \in L(D)$ such that $x, y, z^{*}$ are all distinct. Let $U, V, W$ be disjoint neighborhoods of $x, y$ and $z^{*}$ respectively. By Proposition 2.7 we can find elements $\phi, \psi$ in $D$ such that $\phi$ has fixed points in $U$ and $W$ while $\psi$ has fixed points in $V$ and $W$. Since $U$ and $V$ are disjoint the fixed point sets must be disjoint by Proposition 2.6. Hence either $\phi$ or $\psi$ does not fix $z^{*}$.

A modification of the preceding proof shows that, for any $z \in L(D), D(z)$ is dense in $L(D)$.

The following will be used to prove the theorem about normal coverings stated in the abstract.

Proposition 2.9. Let $N$ be a normal subgroup of $D$. If $L(D)$ has at least three points then $L(N)=L(D)$.

Proof. Clearly $L(N) \subseteq L(D)$. To prove the converse let $x \in L(D)$ be given and fix $\phi \in N$ and $p \in H$. By Proposition 2.6 there exists a point $z \in L(D)$ that is not fixed by $\phi$. There exists a neighborhood $U$ of $z$ such that $\phi(U) \cap U=\square$. If not, then there would exist a sequence $p_{n} \subseteq \bar{H}$ such that $p_{n} \rightarrow z$ and $\phi p_{n} \rightarrow z$. This would imply $z=\phi z$, a contradiction. Let $V$ be a neighborhood of $x$ such that $p \in \bar{H}-V$. By Proposition $2.8 x$ is dual to $z$, hence we may choose $\psi$ in $D$ such that $\psi(\bar{H}-U)$ $\subseteq V$ and $\psi^{-1}(\bar{H}-V) \subseteq U$. Then $p \in \bar{H}-V, \psi^{-1} p \in U, \phi \psi^{-1} p \in \bar{H}-U$ and $\psi \phi \psi^{-1} p$ $\in V$. Since $\psi \phi \psi^{-1} \in N$ and $V$ is arbitrary, $x \in L(N)$.

3. Geodesic flows. In this section we translate our results about $L(D)$ and duality into properties of the geodesic flow on the unit tangent bundle of $M=H / D$. We begin with some general dynamical facts. 
Let $T_{t}$ be a complete flow on a second countable, locally compact, Hausdorff space $X$.

Definition 3.1. For any $v \in X, P^{+}(v)$, the positive prolongational limit set [2] is the set of elements $w \in X$ such that if $O, U$ are any open sets containing $v, w$ respectively then $T_{t}(O)$ meets $U$ for arbitrarily large positive values of $t$.

$P^{+}(v)$ is closed in $X$ and invariant under $T_{t}$. Equivalently $w \in P^{+}(v)$ if and only if there exist a sequence $v_{n} \rightarrow v$ and a sequence of real numbers $t_{n} \rightarrow+\infty$ such that $T_{t_{n}}\left(v_{n}\right) \rightarrow w$.

Definition 3.2. $v \in X$ is nonwandering if $v \in P^{+}(v)$.

Let $\Omega$ denote the set of nonwandering points in $X$. $\Omega$ is closed in $X$ and invariant under $T_{t}$.

Definition 3.3. $T_{t}$ is topologically transitive on $X$ if for some $v \in X$ the orbit $\left\{T_{t}(v): t \in R\right\}$ is dense in $X$.

Proposition 3.4. Suppose that for any $v \in X, P^{+}(v)=X$. Then there exists $z \in X$ such that for any $A>0$ the set $\left\{T_{t}(z): t \geqq A\right\}$ is dense in $X$. In particular $T_{t}$ is topologically transitive on $X$.

Proof. Our assumption says that for any two open sets $O, U$ in $X, T_{t}(O)$ meets $U$ for arbitrarily large positive values of $t$. Let $\left\{O_{i}\right\}$ be a countable basis for the topology of $X$. Fix an open set $O$ in $M$ and choose $t_{1}>1$ such that $T_{t_{1}}(O) \cap O_{1}$ is nonempty. By continuity we can find an open set $A_{1}$ such that $\bar{A}_{1} \subseteq O, \bar{A}_{1}$ is compact and $T_{t_{1}}\left(\bar{A}_{1}\right) \subseteq O_{1}$. Choose $t_{2}>2$ such that $T_{t_{2}}\left(A_{1}\right) \cap O_{2}$ is nonempty and choose an open set $A_{2}$ such that $\bar{A}_{2} \subseteq A_{1}, \bar{A}_{2}$ is compact and $T_{t_{2}}\left(\bar{A}_{2}\right) \subseteq O_{2}$. We define inductively a sequence of open sets $A_{n}$ such that $\bar{A}_{n}$ is compact, $\bar{A}_{n} \subseteq A_{n-1}$ and a sequence $t_{n} \subseteq R$ such that $t_{n}>n$ and $T_{t_{n}}\left(\bar{A}_{n}\right) \subseteq O_{n}$. Since $\bar{A}_{n}$ is a sequence of nested compact sets it has the finite intersection property and $\bigcap_{n=1}^{\infty} \bar{A}_{n}$ is nonempty. If $z$ is in this intersection then $T_{t_{n}}(z) \in O_{n}$ for every $n$. Given an open set $U$ and a number $A>0$ choose $n>A$ such that $O_{n} \subseteq U$. Since $T_{t_{n}}(z) \in O_{n} \subseteq U$ and $t_{n}>n>A$ the result follows.

We now consider the case where $X=S M$, the unit tangent bundle of $M=H / D$, and $T_{t}$ is the geodesic flow on $S M$.

Lemma 3.5. Let $M=H / D$ be a uniform Visibility manifold, $\pi: H \rightarrow M$ the projection. Let unit vectors $v, w \in S M$ have lifts $v^{*}, w^{*}$, unit vectors $\in S H$. Then $w \in P^{+}(v)$ if and only if $\gamma_{v}^{*}(\infty)$ and $\gamma_{w}^{*}(-\infty)$ are dual.

Proof. Suppose that $w \in P^{+}(v)$. Then there exist sequences $v_{n} \rightarrow v$ and $t_{n} \rightarrow+\infty$ such that $T_{t_{n}}\left(v_{n}\right) \rightarrow w$. Let $v^{*}, w^{*}$ be unit vectors in $H$ that are lifts of $v$ and $w$. Choose lifts $v_{n}^{*}$ of $v_{n}$ such that $v_{n}^{*} \rightarrow v^{*}$. If $\gamma_{n}$ is the geodesic with initial velocity $v_{n}^{*}$ then, for some sequence $\phi_{n} \subseteq D,\left(\phi_{n} \circ \gamma_{n}\right)^{\prime}\left(t_{n}\right) \rightarrow w^{*}$. By Proposition 1.15, $\gamma_{n}\left(t_{n}\right)$ $\rightarrow \gamma_{v}^{*}(\infty)$. If $w^{*}$ is tangent to $H$ at $q$ then $d\left(\gamma_{n} t_{n}, \phi_{n}^{-1} q\right) \rightarrow 0$. Hence $\phi_{n}^{-1}(q) \rightarrow \gamma_{v}^{*}(\infty)$. We now show that $\phi_{n}(q) \rightarrow \gamma_{w}^{*}(-\infty)$. By Proposition 2.5 this will show that $\gamma_{v}^{*}(\infty)$ and $\gamma_{w}^{*}(-\infty)$ are dual. Define geodesics $\alpha_{n}: \alpha_{n}(t)=\left(\phi_{n} \circ \gamma_{n}\right)\left(t_{n}-t\right)$. Then 
$\alpha_{n}^{\prime}(0)=-\left(\phi_{n} \circ \gamma_{n}\right)^{\prime}\left(t_{n}\right) \rightarrow-w^{*}$ and $\alpha_{n}\left(t_{n}\right)=\phi_{n} \gamma_{n}(0)$. Let $v^{*}$ be tangent to $H$ at $p$. Since $\alpha_{n}\left(t_{n}\right) \rightarrow \gamma_{w}^{*}(-\infty)$ by Proposition 1.15 and $\gamma_{n}(0) \rightarrow p$ it follows that $\phi_{n}(p)$ $\rightarrow \gamma_{w}^{*}(-\infty)$. By uniform Visibility $\phi_{n}(q) \rightarrow \gamma_{w}^{*}(-\infty)$.

Conversely let $v^{*}, w^{*}$ in $S H$ be lifts of $v, w$ in $S M$ and suppose that $\gamma_{v}^{*}(\infty)$ and $\gamma_{w}^{*}(-\infty)$ are dual. Choose a sequence $\phi_{n} \subseteq D$ such that, for any point $p \in H$, $\phi_{n}^{-1}(p) \rightarrow \gamma_{v}^{*}(\infty)$ and $\phi_{n}(p) \rightarrow \gamma_{w}^{*}(-\infty)$. Let $v^{*}, w^{*}$ be tangent to $H$ at $p, q$ respectively. Let $t_{n}=d\left(\phi_{n} p, q\right)$ and let $\bar{v}_{n}=V\left(\phi_{n} p, q\right)$. Then $T_{t_{n}} \bar{v}_{n}=-V\left(q, \phi_{n} p\right)$ and since $\phi_{n} p \rightarrow \gamma_{w}^{*}(-\infty)$ it follows that $T_{t_{n}} \bar{v}_{n} \rightarrow w^{*}$. Since $\phi_{n}^{-1} q \rightarrow \gamma_{v}^{*}(\infty),\left(\phi_{n}^{-1}\right)_{*} \bar{v}_{n}=$ $V\left(p, \phi_{n}^{-1} q\right) \rightarrow v^{*}$. Then $\pi_{*} \bar{v}_{n}=\pi_{*}\left(\phi_{n}^{-1}\right)_{*} \bar{v}_{n} \rightarrow \pi_{*} v^{*}=v$. Also $T_{t_{n}} \pi_{*} \bar{v}_{n}=\pi_{*} T_{t_{n}} \bar{v}_{n} \rightarrow \pi_{*} w^{*}$ $=w$. Hence $w \in P^{+}(v)$.

Proposition 3.6. Let $M=H / D$ be a uniform Visibility manifold. Then the following are equivalent:

(1) $\Omega=S M$,

(2) $L(D)=H(\infty)$

(3) $P^{+}(v)=S M$ for every $v \in S M$.

Proof. (1) $\Rightarrow(2)$. Let $x \in H(\infty)$ be given and let $\sigma$ be any geodesic such that $\sigma(\infty)=x$. Then $(\pi \circ \sigma)^{\prime}(0)=v \in P^{+}(v)$ by assumption, hence $\sigma(\infty)$ and $\sigma(-\infty)$ are dual by Lemma 3.5. Since dual points must lie in $L(D), x \in L(D)$.

(2) $\Rightarrow(3)$. Let $v, w \in S M$ be given and choose lifts $v^{*}, w^{*}$ in $S H$. Since $\gamma_{v}^{*}(\infty)$ and $\gamma_{w}^{*}(-\infty)$ are in $L(D)$ they are dual by Proposition 2.8. Hence by Lemma 3.5, $w \in P^{+}(v)$.

(3) $\Rightarrow$ (1). This follows immediately.

We may now prove the two main theorems of the abstract.

THEOREM 3.7. Let $M$ be a uniform Visibility manifold such that $\Omega=S M$. Then the geodesic flow is topologically transitive on $S M$.

Proof. This is immediate from Propositions 3.6 and 3.4.

THEOREM 3.8. Let $M$ be a uniform Visibility manifold, $M^{\prime}$ a normal Riemannian covering of $M$. If the geodesic flow on $S M$ is topologically transitive then the geodesic flow on $S M^{\prime}$ is topologically transitive.

Proof. We may write $M=H / D, M^{\prime}=H / N$ where $N$ is a subgroup of $D$. The condition that $M^{\prime}$ be a normal covering of $M$ is the condition that $N$ be a normal subgroup of $D$. Since topological transitivity implies that $\Omega=S M$ it follows from Propositions 2.9 and 3.6 that $L(N)=L(D)=H(\infty) . \Omega=S M^{\prime}$ by Proposition 3.6 and the geodesic flow is topologically transitive on $S M^{\prime}$ by Theorem 3.7.

REMARKS. (1) If $M$ is any compact Riemannian manifold then $\Omega=S M$. To show this let $\mu$ be a volume element in $S M$ that is invariant under the geodesic flow. Suppose that for some open set $O, T_{t}(O) \cap O=\square$ for $t \geqq A>0$. Then the sets $\left\{T_{n A}(O): n \geqq 1\right\}$ are disjoint and the measure of their union is infinite, contradicting the fact that $\mu(S M)$ is finite. 
(2) Theorem 3.8 allows us to construct manifolds of infinite volume for which the geodesic flow is topologically transitive. For example if $M$ is a compact, orientable surface of genus $\geqq 2$ then it admits a metric with $K \equiv-1$. The commutator subgroup of $\pi_{1}(M)$ is a normal subgroup of infinite index hence the normal covering $M^{\prime}$ corresponding to it has infinite volume.

\section{Appendix I.}

THEOREM 4.1. Let $M$ be a compact manifold of $K \leqq 0$. Let $H$ be its simply connected Riemannian covering manifold. Then the Visibility axiom fails in $H$ if and only if $H$ contains a totally geodesic isometric imbedding of the Euclidean plane $R^{2}$.

The proof will also show that this result is true for a homogeneous space $H$. In this section we assume that $H$ has curvature $K \leqq 0$.

LEMma 4.2. Suppose that, for each $T>0, H$ contains a totally geodesic isometric imbedding of a square in $R^{2}$ of side $>T$. Suppose that for some compact set $A$ in $H$ the translates of $A$ by the isometries of $H$ form a covering of $H$. Then $H$ contains a totally geodesic isometric imbedding of $R^{2}$.

Proof. For each positive integer $n$ let $A_{n}$ be a flat totally geodesic square in $H$ of side $t_{n}>n$. Let $p_{n}$ be the center of $A_{n}$ and choose a sequence $\phi_{n} \subseteq D$ such that $q_{n}=\phi_{n} p_{n}$ is a bounded sequence. Let $\pi_{n}$ be the tangent plane to $B_{n}=\phi_{n}\left(A_{n}\right)$ at $q_{n}$ and let $v_{n}, w_{n}$ be orthonormal vectors in $\pi_{n}$. Passing to a subsequence if necessary let $q_{n} \rightarrow q, v_{n} \rightarrow v$ and $w_{n} \rightarrow w$. Let $\pi$ be the plane at $q$ spanned by $v$ and $w$ and let $H_{0}=\exp _{q}(\pi)$. Then $H_{0}$ is the desired flat totally geodesic plane.

Let $H_{n}=\exp _{a_{n}}\left(\pi_{n}\right)$. If $a_{n}$ is any sequence such that $a_{n} \in H_{n}$ and $a_{n} \rightarrow a$ then $a \in H_{0}$ by continuity. Conversely any point in $H_{0}$ is a limit of points in $H_{n}$. Let $p \in H_{0}$ be given. We show that $H_{0}$ is flat and totally geodesic at $p$. Let $p_{n} \in H_{n}$ be chosen so that $p_{n} \rightarrow p$. Let $x_{n}, y_{n}$ be orthonormal vectors in the tangent plane of $H_{n}$ at $p_{n}$. For sufficiently large $n, 0 \leqq \theta<2 \pi,-1 \leqq t \leqq 1$, the vectors $\cos \theta x_{n}+\sin \theta y_{n}$ are tangent to $B_{n}$ and $\exp _{p_{n}}\left(t \cos \theta x_{n}+t \sin \theta y_{n}\right) \in B_{n}$ since $B_{n}$ is totally geodesic. Passing to a subsequence let $x_{n} \rightarrow x, y_{n} \rightarrow y$ in $H_{p}$. By continuity

$$
\exp _{p}(t \cos \theta x+t \sin \theta y) \in H_{0} \text { for }-1 \leqq t \leqq 1,0 \leqq \theta<2 \pi \text {. }
$$

The vectors $\cos \theta x+\sin \theta y$ are therefore tangent to $H_{0}$ at $p . H_{0}$ is totally geodesic at $p$ since it contains an initial geodesic segment in every direction. It suffices to prove that $\bar{K}(\sigma)=0$ where $\sigma$ is the tangent plane of $H_{0}$ at $p$ and $\bar{K}$ denotes sectional curvature in the containing manifold $H$. If $\sigma_{n}$ is the plane spanned by $x_{n}$ and $y_{n}$ then $\bar{K}\left(\sigma_{n}\right)=0$ since $B_{n}$ is flat. Thus $\bar{K}(\sigma)=0$ by continuity.

Lemma 4.3. Suppose that the Visibility axiom fails in $H$. Then there exist distinct points $x, y$ in $H(\infty)$ such that $\chi_{p}(x, y) \leqq \pi / 2$ for every point $p$ in $H$.

Proof. If the Visibility axiom fails then there exists an $\varepsilon>0$, a point $p \in H$ and a sequence of geodesic segments $\sigma_{n}:\left[a_{n}, b_{n}\right] \rightarrow H$ such that $\chi_{p}\left(\sigma_{n} a_{n}, \sigma_{n} b_{n}\right) \geqq \varepsilon>0$ 
but $d\left(p, \sigma_{n}\right) \rightarrow \infty$. Let $r_{n}$ be the point on $\sigma_{n}$ closest to $p$. Passing to a subsequence if necessary either $\chi_{p}\left(r_{n}, \sigma_{n} b_{n}\right) \geqq \varepsilon / 2$ for all $n$ or $\chi_{p}\left(r_{n}, \sigma_{n} a_{n}\right) \geqq \varepsilon / 2$ for all $n$. For simplicity we assume that the first case occurs. By Hadamard trigonometry spheres are convex and hence $\chi_{r_{n}}\left(p, \sigma_{n} b_{n}\right) \geqq \pi / 2$. Passing to a further subsequence let $r_{n} \rightarrow x$ and $\sigma_{n} b_{n} \rightarrow y$. By the continuity of angle measurement $\chi_{p}(x, y) \geqq \varepsilon / 2$ so $x \neq y$. Let $q \in H$ and $\delta>0$ be given. By the law of cosines $\chi_{r_{n}}(q, p) \rightarrow 0$ hence for sufficiently large $n, \chi_{r_{n}}(q, p) \leqq \delta$. The sum of the angles in a geodesic triangle of $H$ is $\leqq \pi$. This is true even if one of the vertices lies in $H(\infty)$, that is if two of the bounding geodesics are asymptotes [3]. If $\chi_{r_{n}}(q, p) \leqq \delta$ then $\chi_{r_{n}}\left(q, \sigma_{n} b_{n}\right) \geqq \pi / 2-\delta$ and it follows that $\chi_{q}\left(r_{n}, \sigma_{n} b_{n}\right) \leqq \pi / 2+\delta$. By continuity $\chi_{q}(x, y) \leqq \pi / 2+\delta$. Since $\delta>0$ was arbitrary $\chi_{q}(x, y) \leqq \pi / 2$.

We now prove Theorem 4.1. If $H$ contains a flat totally geodesic plane then the Visibility axiom clearly fails in $H$ since it fails in any Euclidean space. Conversely suppose that the Visibility axiom fails. We shall need the following fact from [3]. Let $\gamma, \sigma$ be asymptotes such that $d(\sigma t, \gamma)=d(\gamma t, \sigma) \equiv c>0$ for all $t \geqq 0$. Then there exists a totally geodesic isometric imbedding $x:[0, \infty) \times[0, c] \rightarrow H$ such that $x(t, 0)=\gamma(t)$ and $x(t, c)=\sigma(t)$. Briefly, $\gamma$ and $\sigma$ bound a flat half strip. By Lemma 4.3 we may choose distinct points $x, y$ such that $\chi_{p}(x, y) \leqq \pi / 2$ for every point $p$. Let $\theta=\sup \left\{\chi_{p}(x, y): p \in H\right\}$ and let $p_{n}$ be a sequence in $H$ such that $\chi_{p_{n}}(x, y)$ $\rightarrow \theta$. Choose a sequence $\phi_{n}$ in $D$ such that $q_{n}=\phi_{n} p_{n}$ is a bounded sequence. Passing to a subsequence let $q_{n} \rightarrow q, \phi_{n} x \rightarrow x^{*}$ and $\phi_{n} y \rightarrow y^{*} . \chi_{a_{n}}\left(\phi_{n} x, \phi_{n} y\right)=\chi_{p_{n}}(x, y)$ hence by continuity $\chi_{q}\left(x^{*}, y^{*}\right)=\theta>0$. Furthermore $\chi_{p}\left(x^{*}, y^{*}\right) \leqq \theta$ for any point $p \in H$; if there existed $p \in H$ such that $\chi_{p}\left(x^{*}, y^{*}\right)>\theta$ then, for sufficiently large $n$, $\chi_{\varphi_{n}^{-1} p}(x, y)=\chi_{p}\left(\phi_{n} x, \phi_{n} y\right)>\theta$, which contradicts the definition of $\theta$. Let $\sigma_{1}$ be the geodesic ray joining $q$ to $x^{*}, \sigma_{2}$ the geodesic ray joining $q$ to $y^{*}$. We show essentially that $\sigma_{1}$ and $\sigma_{2}$ bound a flat triangular sector. Fix $t>0$. By the remark above, $\chi_{\sigma_{1} t}\left(x^{*}, y^{*}\right) \leqq \theta$. By the angle sum relation for infinite triangles [3], $\chi_{\sigma_{1} t}\left(q, y^{*}\right)$ $\leqq \pi-\theta$ and hence $\chi_{\sigma_{1} t}\left(x^{*}, y^{*}\right) \geqq \theta$. Thus $\chi_{\sigma_{1} t}\left(x^{*}, y^{*}\right)=\theta$ and $\chi_{\sigma_{1} t}\left(q, y^{*}\right)=\pi-\theta$. Let $q_{t}$ be the foot of $\sigma_{1} t$ on the maximal geodesic extension of $\sigma_{2}$. Since $\theta \leqq \pi / 2, q_{t}$ $=\sigma_{2} s$ for $s \geqq 0$.

We show that $\chi_{\sigma_{1} t}\left(q_{t}, y^{*}\right)=\pi / 2$. If $s=0$ then $q_{t}=q, \theta=\pi / 2$ and $\chi_{\sigma_{1} t}\left(q, y^{*}\right)$ $=\pi-\theta=\pi / 2$. Suppose $s>0$. By the angle sum relation, $\chi_{\sigma_{1} t}\left(q, q_{t}\right) \leqq \pi / 2-\theta$. Hence

$$
\pi-\theta=\chi_{\sigma_{1} t}\left(q, y^{*}\right) \leqq \chi_{\sigma_{1} t}\left(q, q_{t}\right)+\chi_{\sigma_{1} t}\left(q_{t}, y^{*}\right) \leqq \pi / 2-\theta+\chi_{\sigma_{1} t}\left(q_{t}, y^{*}\right) .
$$

Hence $\chi_{\sigma_{1} t}\left(q_{t}, y^{*}\right) \geqq \pi / 2$. Since $\chi_{q_{t}}\left(\sigma_{1} t, y^{*}\right)=\pi / 2$ the angle sum relation implies that $\chi_{\sigma_{1} t}\left(q_{t}, y^{*}\right) \leqq \pi / 2$. Therefore $\chi_{\sigma_{1} t}\left(q_{t}, y^{*}\right)=\pi / 2$ and the geodesic from $q_{t}$ to $\sigma_{1} t$ is perpendicular to both $\sigma_{2}$ and the geodesic $\rho$ from $\sigma_{1} t$ to $y^{*}$. Let $C_{t}=d\left(\sigma_{1} t, q_{t}\right)$. Since the transversal realizes the distance from $\rho$ to $\sigma_{2}$ it follows that $d\left(\rho s, \sigma_{2}\right) \geqq C_{t}$ for $s \geqq 0$. Since $\rho$ and $\sigma_{2}$ are asymptotes, $s \rightarrow d\left(\rho s, \sigma_{2}\right)$ is a bounded convex function for $s \geqq 0$ and hence is monotone decreasing [3]. Therefore $d\left(\rho s, \sigma_{2}\right) \equiv C_{t}$ for $s \geqq 0$ and, by the fact mentioned previously, $\rho$ and $\sigma_{2}$ bound a flat half strip of width $C_{t}$. 
Since $t>0$ was arbitrary, $C_{t} \rightarrow \infty$ as $t \rightarrow \infty$ and we may find a flat totally geodesic square of arbitrarily large side in $H$. By Lemma $4.2, H$ contains a totally geodesic isometric imbedding of the plane.

COROLlary 4.4. Let $M$ be a compact manifold with curvature $K<0$. Then $M$ is a uniform Visibility manifold.

Proof. $M$ is a Visibility manifold by Theorem 4.1 . Since $M$ is compact $M$ is also uniform by the remarks in $\S 1$.

5. Appendix II. In the present section we derive a sufficient condition for manifolds without conjugate points to satisfy the uniform Visibility axiom. Our main result is the following:

THEOREM 5.1 $\left({ }^{4}\right)$. Let $M$ be a compact uniform Visibility manifold with metric $g$. Let $g^{*}$ be any metric on $M$ that admits no conjugate points. Then $M$ with the metric $\mathrm{g}^{*}$ is also a uniform Visibility manifold. In particular the $\mathrm{g}^{*}$-geodesic flow is topologically transitive on the $g^{*}$ unit tangent bundle.

COROLlaRY $5.2\left({ }^{5}\right)$. Let $M$ be a compact Visibility manifold with metric $g$ and $K \leqq 0$. Let $g^{*}$ be any metric on $M$ without conjugate points. Then the $g^{*}$-geodesic flow is topologically transitive on the $g^{*}$ unit tangent bundle.

Corollary 5.2 is proved in [8] for the case $K<0$.

The method of this section is based on an idea of Morse [9] that was later extended by Klingenberg [8]. One shows that if $\gamma, \gamma^{*}$ are geodesics with the same endpoints in the metrics $g, g^{*}$ in the simply connected covering $H$, then $\gamma$ and $\gamma^{*}$ cannot spread apart farther than a certain universal constant $R$. This statement is made precise in Proposition 5.5.

Definition 5.3. Metrics $g, g^{*}$ on a Riemannian manifold $M$ are equivalent if there exist constants $0<a \leqq b$ such that for any vector $v$ tangent to $M$

$$
a\|v\| \leqq\|v\|_{*} \leqq b\|v\|
$$

where \|\|$,\|\|_{*}$ denote norms with respect to $g, g^{*}$.

Clearly if $M$ is compact then any two metrics on $M$ are equivalent.

Proposition 5.4. Let $g, g^{*}$ be equivalent metrics on a Riemannian manifold $M$. If $a\|v\| \leqq\|v\|_{*} \leqq b\|v\|$ for all tangent vectors $v$ then

(1) $a L(\sigma) \leqq L^{*}(\sigma) \leqq b L(\sigma)$ for any $C^{\infty}$ curve $\sigma$ in $H$.

(2) $\operatorname{ad}(P, Q) \leqq d^{*}(P, Q) \leqq b d(P, Q)$ for any points $P, Q$ in $H$.

(3) $g$ is complete if and only if $g^{*}$ is complete.

Proof. $L, d$ and $L^{*}, d^{*}$ denote length and distance with respect to $g$ and $g^{*}$. (1) and (2) are straightforward and (3) follows from (2) using the theorem of Hopf and Rinow.

(4) See Added in proof.

(5) See Added in proof. 
Proposition 5.5. Let $H$ be a simply connected uniform Visibility manifold with metric $g$. Let $g^{*}$ be an equivalent metric on $H$ (which may admit conjugate points). Then there exists a constant $R>0$ with the following property: if $\gamma, \gamma^{*}$ are geodesic segments in the metrics $g, g^{*}$ with the same endpoints and if $\gamma^{*}$ is ( $g^{*}$-distance) minimizing then for any point $x \in \gamma, d\left(x, \gamma^{*}\right) \leqq R$, where $d$ is the distance with respect to the metric $g$.

The existence of this universal constant was proved in [9] for the case where $H$ is the hyperbolic plane with the Poincare metric and in [8] for the case where $H$ has arbitrary dimension and $K \leqq c<0$. We break up the proof into a series of lemmas, assuming in all of them that $H$ is a simply connected uniform Visibility manifold.

LEMMA 5.6. There exists a constant $A>0$ such that if $T$ is a geodesic right triangle in $H$ with a leg of length $\geqq A$ then the base angle corresponding to this leg is $\leqq \pi / 4$.

Proof. Let $B>0$ be chosen so that for any point $p$ and geodesic $\gamma, d(p, \gamma) \leqq B$ if $\chi_{p}(\gamma) \geqq \pi / 2$. By uniform Visibility we may choose $A>0$ so that if $p, q, a$ are points satisfying $d(q, p) \geqq A$ and $d(p, a) \leqq B$ then $\chi_{q}(p, a) \leqq \pi / 4$. Let $T$ be a geodesic right triangle with vertices $p, q, r$, hypotenuse $\gamma$ opposite $p$ and leg $p q$ of length $\geqq A$. There exists $a \in \gamma$ such that $d(p, a) \leqq B$ and hence $\chi_{q}(p, r)=\chi_{q}(p, a) \leqq \pi / 4$.

LEMMA 5.7. For any constant $C>0$ there exists a constant $R=R(C)>0$ such that the following is true: if $p$ is any point in $H$ and if $\sigma:[a, b] \rightarrow H$ is any $C^{\infty}$ curve satisfying $d(p, \sigma) \geqq R$ and $\chi_{p}(\sigma a, \sigma b) \geqq \pi / 2$ then $L(\sigma) \geqq C R$.

Proof. Given $C>0$ choose an integer $n>0$ such that $C<2 n$. Choose $R$ so large that if $\alpha$ is a geodesic segment subtending an angle $\geqq \pi / 2 n$ at $p$ then $d(p, \alpha)$ $<R(1-(C / 2 n))$. $R$ can be chosen independent of the point $p$ since $H$ is uniform. Let a point $p$ in $H$ be given and let $\sigma:[a, b] \rightarrow H$ be a $C^{\infty}$ curve such that $d(p, \sigma) \geqq R$ and $\chi_{p}(\sigma a, \sigma b)=\delta \geqq \pi / 2$. By the continuity of angle measurement we may find a sequence $a=t_{0}<t_{1}<\cdots<t_{n}=b$ such that

$$
\chi_{p}\left(\sigma a, \sigma t_{i}\right)=i \delta / n \text { for } 1 \leqq i \leqq n .
$$

Then

$$
\chi_{p}\left(\sigma t_{i-1}, \sigma t_{i}\right) \geqq \chi_{p}\left(\sigma a, \sigma t_{i}\right)-\chi_{p}\left(\sigma a, \sigma t_{i-1}\right)=\delta / n \geqq \pi / 2 n .
$$

If $\alpha_{i}$ is the geodesic segment joining $\sigma t_{i-1}$ to $\sigma t_{i}$ then $d\left(p, \alpha_{i}\right)<R(1-(C / 2 n))<R$ since $\alpha_{i}$ subtends an angle $\geqq \pi / 2 n$ at $p$. A point on $\alpha_{i}$ closest to $p$ must be an interior point since $d\left(p, \alpha_{i}\right)<R$. It follows that $L\left(\alpha_{i}\right) \geqq 2\left(R-d\left(p, \alpha_{i}\right)\right) \geqq C R / n$. Hence $L(\sigma) \geqq \sum_{i=1}^{n} L\left(\alpha_{i}\right) \geqq C R$.

LEMMA 5.8. For any constant $C>0$ there exists a constant $R=R(C)>0$ such that the following is true: Let $\gamma$ be a maximal geodesic of $H$ and let $\sigma:[a, b] \rightarrow H-\gamma$ be a $C^{\infty}$ curve. Let Poa, Pob denote the feet on $\gamma$ of perpendiculars to $\gamma$ from $\sigma a$ and $\sigma b$ respectively. Then if $d(\sigma, \gamma) \geqq R$ and $d(P \sigma a, P \sigma b) \geqq 2 A$ then $L(\sigma) \geqq C R d(P \sigma a, P \sigma b)$. 
Proof. $A$ is the constant of Lemma 5.6. Let $R=R(10 A C)$ in the notation of Lemma 5.7. Let $\sigma:[a, b] \rightarrow H$ and $\gamma$ satisfy the hypotheses above. We first consider the case where $2 A \leqq d(P \sigma a, P \sigma b) \leqq 4 A$. Let $q$ be the midpoint of the segment from $P \sigma a$ to $P \sigma b$. By Lemma 5.6, $\chi_{q}(P \sigma a, \sigma a) \leqq \pi / 4, \chi_{q}(P \sigma b, \sigma b) \leqq \pi / 4$ and hence $\chi_{q}(\sigma a, \sigma b) \geqq \pi / 2$. Since $d(q, \sigma) \geqq R$, by Lemma 5.7, L( $\left.\sigma\right) \geqq 10 A C R \geqq C R d(P \sigma a, P \sigma b)$. Suppose now that $d(P \sigma a, P \sigma b) \geqq 4 A$ and let $k \geqq 2$ be the integer such that

$$
k \leqq d(P \sigma a, P \sigma b) / 2 A \leqq k+1 .
$$

Let $P \sigma a=x_{0}, x_{1} \cdots x_{k-1}$ be a linearly ordered sequence of points on the segment of $\gamma$ between $P \sigma a$ and $P \sigma b$ such that $d\left(x_{i}, x_{i-1}\right)=2 A$ for every $i=0,1, \ldots, k-1$. Inductively we shall construct a sequence $a=t_{0}<t_{1}<\cdots<t_{k-1}<b$ such that the geodesic from $\sigma\left(t_{i}\right)$ to $x_{i}$ is perpendicular to $\gamma$ for $i=1, \ldots, k-1$. Since $d\left(x_{1}, x_{0}\right)$ $=2 A$ and $d\left(x_{1}, P \sigma b\right) \geqq 2 A$, Lemma 5.6 implies that $\chi_{x_{1}}\left(x_{0}, \sigma a\right) \leqq \pi / 4$ and $\chi_{x_{1}}\left(x_{0}, \sigma b\right) \geqq 3 \pi / 4$. By continuity there exists $t_{1}: a<t_{1}<b$ and the geodesic from $\sigma\left(t_{1}\right)$ to $x_{1}$ is perpendicular to $\gamma$. Suppose that $t_{i-1}$ has been constructed. Then $d\left(x_{i}, x_{i-1}\right)=2 A$ and $d\left(x_{i}, P \sigma b\right) \geqq 2 A$ hence $\chi_{x_{i}}\left(x_{i-1}, \sigma t_{i-1}\right) \leqq \pi / 4$ and $\chi_{x_{i}}\left(x_{i-1}, \sigma b\right)$ $\geqq 3 \pi / 4$. As before we may construct $t_{i}$ with the required properties. Let $\sigma_{i}$ denote the restriction of $\sigma$ to $\left[t_{i-1}, t_{i}\right]$ for $i=1, \ldots, k-1$. By the argument above $L\left(\sigma_{i}\right)$ $\geqq 10 A C R$ for every $i$ hence $L(\sigma) \geqq \sum_{i=1}^{k-1} L\left(\sigma_{i}\right) \geqq(k-1) 10 A C R \geqq C R d(P \sigma a, P \sigma b)$ since $k \geqq 2$.

Let $\gamma, \sigma:[a, b] \rightarrow H-\gamma$ be defined as above. However we now assume that Poa, $P \sigma b$ are points on $\gamma$ closest to $\sigma a, \sigma b$ respectively. Hence $d(\sigma a, \gamma)=d(\sigma a, P \sigma a)$ and $d(\sigma b, \gamma)=d(\sigma b, P \sigma b)$.

LEMMA 5.9. For any constant $B>0$ there exists a constant $R=R(B)>0$ such that if

(1) $d(\sigma, \gamma) \geqq R$,

(2) $d(\sigma a, \gamma)=d(\sigma b, \gamma)=R$,

(3) $d(P \sigma a, P \sigma b) \geqq 2 A$,

then

$$
L(\sigma) \geqq B d(\sigma a, \sigma b) .
$$

Proof. Given $B>0$ choose $C>0$ so that

$$
1 / C A+1 / C<1 / B
$$

Choose $R=R(C) \geqq 1$ as in Lemma 5.8. Then if $D=d(P \sigma a, P \sigma b)$,

$$
d(\sigma a, \sigma b) / L(\sigma) \leqq(2 R+D) / C R D=2 / D C+1 / C R \leqq 1 / C A+1 / C<1 / B .
$$

Hence

$$
L(\sigma)>B d(\sigma a, \sigma b) .
$$

LEMMA 5.10. Let $H$ be a uniform Visibility manifold with metric $g$. Let $g^{*}$ be an equivalent metric and let $0<a \leqq b$ be constants such that $a\|v\| \leqq\|v\|_{*} \leqq b\|v\|$ for all 
tangent vectors $v$. Let $\gamma$ be a maximal g-geodesic, $\gamma^{*}:[c, d] \rightarrow H$ a minimizing $g^{*}$-geodesic. Choose $B>b / a$ and let $R=R(B)$ be the constant of Lemma 5.9. Suppose that $d\left(\gamma^{*}, \gamma\right) \geqq R$ and $d\left(\gamma^{*} c, \gamma\right)=d\left(\gamma^{*} d, \gamma\right)=R$. Then $d\left(P \gamma^{*} c, P \gamma^{*} d\right)<2 A$.

Proof. Suppose that $d\left(P \gamma^{*} c, P \gamma^{*} d\right) \geqq 2 A$. By Lemma 5.9 and Proposition 5.4

$$
L^{*}\left(\gamma^{*}\right) \geqq a L\left(\gamma^{*}\right) \geqq a B d\left(\gamma^{*} c, \gamma^{*} d\right) \geqq(a \mid b) B d^{*}\left(\gamma^{*} c, \gamma^{*} d\right)>d^{*}\left(\gamma^{*} c, \gamma^{*} d\right) .
$$

This contradicts the assumption that $\gamma^{*}$ is minimizing in the metric $g^{*}$.

Lemma 5.11. Let $g, g^{*}, a, b$ and $R$ be as in Lemma 5.10. Let $R^{*}=R(1+(2 b / a))$ $+2 b A / a$. Let $\gamma$ be a maximal g-geodesic, $\gamma^{*}:[c, d] \rightarrow H$ a minimizing $g^{*}$-geodesic whose endpoints lie on $\gamma$. Then $d(x, \gamma) \leqq R^{*}$ for any point $x$ on $\gamma^{*}$.

Proof. Suppose that there exists $x \in \gamma^{*}$ such that $d(x, \gamma)>R$; we show that $d(x, \gamma) \leqq R^{*}$. Let $y, z$ be the first points on $\gamma^{*}$ on either side of $x$ such that $d(y, \gamma)$ $=d(z, \gamma)=R$. If $P y, P z$ denote points on $\gamma$ that are closest to $y, z$ then, by Lemma 5.10, $d(P y, P z)<2 A$ since $\gamma^{*}$ is minimizing. Thus $d(y, z) \leqq 2 R+2 A$. Now $d(x, y)$ $\leqq g$-length of $\gamma^{*}$ between $y$ and $z \leqq(1 / a) d^{*}(y, z) \leqq(b / a) d(y, z) \leqq(b / a)(2 R+2 A)$. Hence $d(x, \gamma) \leqq R+d(x, y) \leqq R+(b / a)(2 R+2 A)=R^{*}$.

We now complete the proof of Proposition 5.5. Let $R^{*}$ be the constant of Lemma 5.11, $A$ the constant of Lemma 5.6. Then $R=R^{*}+A$ is the desired universal constant. Let $\gamma, \gamma^{*}$ be geodesics with the same endpoints as in the statement of Proposition 5.5. Suppose that there is a point $x$ on $\gamma$ such that $d\left(x, \gamma^{*}\right)>R$. Let $\gamma:[a, b]$ $\rightarrow H$ be a unit speed parametrization of $\gamma$ with $x=\gamma t_{0}, a<t_{0}<b$, and extend $\gamma$ to be maximal. Let $O=\left\{y \in \gamma^{*}\right.$ : for some $-\infty<t<t_{0}, \gamma t$ is a closest point for $\left.y\right\}$. Let $U=\left\{y \in \gamma^{*}\right.$ : for some $t_{0}<t<\infty, \gamma t$ is a closest point for $\left.y\right\}$. We show that $O$ and $U$ are a separation for $\gamma^{*}$, contradicting the connectedness of $\gamma^{*}$.

If $\gamma t, \gamma t^{\prime}$ are two closest points in $\gamma$ for a point $y$ in $\gamma^{*}$ then, by Lemma 5.6, $d\left(\gamma t, \gamma t^{\prime}\right) \leqq A$. Since $d(x, y)>R$ and $d(y, \gamma t)=d\left(y, \gamma t^{\prime}\right) \leqq R^{*}$ we see that $d(x, \gamma t)$ $>A$ and $d\left(x, \gamma t^{\prime}\right)>A$. It follows that $O$ and $U$ are nonempty, disjoint sets whose union is $\gamma^{*}$. If $O$ were not open there would exist $y \in O$ and a sequence $y_{n} \subseteq U$ such that $y_{n} \rightarrow y$. If $\gamma t, \gamma t_{n}$ are closest points for $y, y_{n}$ respectively then $\left|t-t_{n}\right| \geqq 2 A$ for every $n$ since $t<t_{0}<t_{n},\left|t-t_{0}\right| \geqq A$ and $\left|t_{0}-t_{n}\right| \geqq A$. The points $y_{n}, \gamma t_{n}$ and $\gamma t$ determine a $g$-geodesic triangle with a right angle at $\gamma t_{n}$. Since $y_{n} \rightarrow y$ the angle at $\gamma t \rightarrow \pi / 2$, contradicting Lemma 5.6. Hence $O$ is open. Similarly $U$ is open, proving that $O$ and $U$ are a separation for $\gamma^{*}$. This contradiction completes the proof of Proposition 5.5.

We now prove Theorem 5.1. Let $g, g^{*}$ also denote the metrics induced in the simply connected covering $H$. Let $D$ be the deckgroup of $M$ such that $M=H / D$. Suppose $g^{*}$ is a metric without conjugate points that does not satisfy the uniform Visibility axiom. Then for some $\varepsilon>0$ there exist a sequence $p_{n} \subseteq H$ and a sequence $\sigma_{n}^{*}:\left[a_{n}, b_{n}\right] \rightarrow H$ of $g^{*}$-geodesic segments such that $\chi_{p_{n}}^{*}\left(\sigma_{n}^{*} a_{n}, \sigma_{n}^{*} b_{n}\right) \geqq \varepsilon>0$ for all $n$ but $d^{*}\left(p_{n}, \sigma_{n}^{*}\right) \rightarrow \infty$. Choose a sequence $\phi_{n} \subseteq D$ such that $q_{n}=\phi_{n} p_{n}$ is bounded 
and, passing to a subsequence if necessary, let $q_{n} \rightarrow q$. If $\gamma_{n}^{*}=\phi_{n} \circ \sigma_{n}^{*}$ then

$$
\chi_{a_{n}}^{*}\left(\gamma_{n}^{*} a_{n}, \gamma_{n}^{*} b_{n}\right) \geqq \varepsilon>0
$$

and $d^{*}\left(q_{n}, \gamma_{n}^{*}\right) \rightarrow \infty$ since the elements of $D$ are also isometries in the metric $g^{*}$. Let $\alpha_{n}^{*}, \beta_{n}^{*}$ be the $g^{*}$-geodesics from $q_{n}$ to $\gamma_{n}^{*} a_{n}$ and from $q_{n}$ to $\gamma_{n}^{*} b_{n}$ respectively. Let $\alpha_{n}, \beta_{n}$ be the corresponding $g$-geodesics with the same endpoints and let $\gamma_{n}$ be the $g$-geodesic from $\gamma_{n}^{*} a_{n}$ to $\gamma_{n}^{*} b_{n}$. By Proposition 5.5 and the equivalence of $g$ and $g^{*}$, $d\left(q_{n}, \gamma_{n}\right) \rightarrow \infty$. Measuring with respect to $g, \chi_{a_{n}}\left(\gamma_{n}^{*} a_{n}, \gamma_{n}^{*} b_{n}\right) \rightarrow 0$ since $H$ with the metric $g$ is a uniform Visibility manifold. With initial point $q$ there exist a $g$-geodesic ray $\alpha$ and $g^{*}$-geodesic rays $\alpha^{*}, \beta^{*}$ such that, passing to a subsequence if necessary, $\alpha_{n}^{\prime}(0) \rightarrow \alpha^{\prime}(0), \beta_{n}^{\prime}(0) \rightarrow \alpha^{\prime}(0), \alpha_{n}^{* \prime}(0) \rightarrow \alpha^{*^{\prime}}(0)$ and $\beta_{n}^{* \prime}(0) \rightarrow \beta^{* \prime}(0)$. By hypothesis and continuity $\alpha^{*} \neq \beta^{*}$. If $x$ is any point on $\alpha$ then we can find $x_{n}$ on $\alpha_{n}$ such that $x_{n} \rightarrow x$. Since $d\left(x_{n}, \alpha_{n}^{*}\right) \leqq R$ by Proposition 5.5 and $\alpha_{n}^{* \prime}(0) \rightarrow \alpha^{*^{\prime \prime}}(0)$ it follows that $d\left(x, \alpha^{*}\right) \leqq R$. Similarly $d\left(x, \beta^{*}\right) \leqq R$. Let $\alpha^{*}:[0, \infty) \rightarrow H$ and $\beta^{*}:[0, \infty) \rightarrow H$ be $g^{*}$ unit speed parametrizations of $\alpha^{*}$ and $\beta^{*}$. For each integer $n>0$ let numbers $s_{n}$ and $t_{n}$ be chosen so that $d\left(\alpha^{*} s_{n}, \alpha(n)\right) \leqq R$ and $d\left(\beta^{*} t_{n}, \alpha(n)\right) \leqq R$. Then $s_{n} \rightarrow \infty$ and $d^{*}\left(\alpha^{*} s_{n}, \beta^{*}\right) \leqq b d\left(\alpha^{*} s_{n}, \beta^{*}\right) \leqq 2 b R$. A result of Green [4], however, states that if $\alpha^{*}, \beta^{*}$ are distinct geodesic rays with the same initial point in a simply connected manifold $H$ without conjugate points and with curvature $K \geqq-A^{2}$ then $d^{*}\left(\alpha^{*} t, \beta^{*}\right) \rightarrow \infty$ as $t \rightarrow \infty$. This contradiction proves that the uniform Visibility axiom holds for $g^{*}$. The topological transitivity assertion follows from Theorem 3.7.

Corollary 5.2 follows immediately from Theorem 5.1 since by the remarks in $\S 1$ Visibility and uniform Visibility are equivalent in a compact manifold with $K \leqq 0$.

Added in proof. The divergence condition for geodesics in $H$, due to L. Green and stated above, is crucial for the argument of Theorem 5.1. For surfaces there is a simpler proof of the divergence condition that does not generalize to higher dimensions [5, p. 534]. The proof for higher dimensions is a consequence of Lemma 2 in [4], but the proof seems to us incomplete. If $M$ has dimension $n \geqq 3$ it may therefore be necessary in Theorem 5.1 and Corollary 5.2 to consider only those auxiliary metrics without conjugate points that satisfy the divergence condition for geodesics in the simply connected covering $H$. In particular the manifolds without focal points and curvature $K \geqq-A^{2}$ satisfy this condition, and we now sketch a proof.

Lemma 2 in [4] is essentially equivalent to the following uniform divergence condition for Jacobi fields: Let $M$ be a complete manifold of dimension $n \geqq 2$ without conjugate points and with curvature $K \geqq-A^{2}$. For each number $R>0$ and each point $p \in M$ we can find a number $t_{0}=t_{0}(p)>0$ such that $\|Y(t)\| \geqq R$ for $t \geqq t_{0}$, where $Y(t)$ is a Jacobi field on a unit speed geodesic $\gamma$ such that $\gamma(0)=p$, $Y(0)=0,\left\|Y^{\prime}(0)\right\|=1$ and $\left\langle Y(t), \gamma^{\prime}(t)\right\rangle=0$ for all $t \geqq 0$. The divergence condition for geodesics in $H$ is an easy consequence of this condition on the Jacobi fields of $M$ (and $H$ ). A complete manifold $M$ is said to have no focal points if no maximal geodesic of $M$ has focal points. It is not difficult to show that the no focal point 
property is equivalent to the following: Let $Z(t)$ be a not necessarily perpendicular Jacobi field on a unit speed geodesic $\gamma$ such that $Z(0)=0$ and $Z^{\prime}(0) \neq 0$. Then $\left\{\|Z\|^{2}\right\}^{\prime}(t)>0$ for every $t>0$. A manifold without focal points clearly has no conjugate points, and the uniform divergence condition for Jacobi fields will follow if we can show that $\|Y(t)\| \rightarrow \infty$ as $t \rightarrow \infty$ for any Jacobi field $Y$ of the type considered in the statement of the condition. To establish this we mimic the proof of Theorem 2.1 of [5] while using the following matrix method described in detail in [6].

Let $M$ be a complete manifold of dimension $n \geqq 2$ without conjugate points and with curvature $K \geqq-A^{2}$. Fix a geodesic $\gamma$ and a Fermi coordinate system along $\gamma$. The Jacobi equations for vector fields perpendicular to $\gamma$ may be transformed into the following $(n-1) \times(n-1)$ matrix equation in one real variable:

$$
Y^{\prime \prime}(s)+K(s) Y(s)=0 .
$$

Derivatives are taken componentwise and $K(s)$ is a symmetric matrix corresponding to the curvature transformation $R(s): M_{\gamma(s)} \rightarrow M_{\gamma(s)}, R(s) v=R_{\gamma^{\prime}(s) v} \gamma^{\prime}(s)$. If $A(s)$ is the solution of $(J)$ such that $A(0)=0, A^{\prime}(0)=$ identity, then the Jacobi fields of the type considered in the statement of the uniform divergence condition correspond, relative to the Fermi coordinate system, to the curves $s \rightarrow A(s) x$, where $x$ is a unit vector in $R^{n-1}$. Let $D(s)$ be the limit solution of $(J)$ defined in Lemma 1 of [6]. Then for $s>0, D(s)=A(s) M(s)$, where $M(s)=\int_{s}^{\infty} A^{-1}(u)\left[A^{-1}(u)\right]^{*} d u$ is a positive definite symmetric matrix. * denotes the transpose operation. $D(s)$ and $A(s)$ are both nonsingular for $s>0$, and we consider the symmetric matrices $U(s)=$ $D^{\prime}(s) D^{-1}(s)$ and $W(s)=A^{\prime}(s) A^{-1}(s)$, which satisfy a matrix Riccati equation obtained from $(J)$. Combining the proofs of Lemma 2.1 of [5] and Lemma 3 of [6] we can choose a number $s_{0}>0$ so that $\|U(s)\|_{\infty}$ and $\|W(s)\|_{\infty}$ are uniformly bounded for $s \geqq s_{0}$, where $\|\cdot\|_{\infty}=\sup \{\|\cdot(x)\|:\|x\|=1\}$. A Wronskian argument shows that $U(s)-W(s)=\left[A^{-1}(s)\right]^{*} M^{-1}(s) A^{-1}(s)$, and hence $\mid\left\langle M^{-1}(s) A^{-1}(s) x\right.$, $\left.A^{-1}(s) x\right\rangle \mid$ is uniformly bounded for $s \geqq s_{0}$ and every unit vector $x \in R^{n-1}$. Since $M(s) \rightarrow 0$ as $s \rightarrow \infty$ and $M^{-1}(s)$ is positive definite and symmetric it follows that $\left\|A^{-1}(s)\right\|_{\infty} \rightarrow 0$ as $s \rightarrow \infty$. Hence for any unit vector $x \in R^{n-1}\|A(s) x\| \geqq$ $1 /\left\|A^{-1}(s)\right\|_{\infty} \rightarrow \infty$ as $s \rightarrow \infty$, which completes the proof.

\section{BIBLIOGRAPHY}

1. D. V. Anosov, Geodesic flows on closed Riemannian manifolds with negative curvature, Proc. Steklov Institute Math., No. 90, Amer. Math. Soc., Providence, R. I., 1969. MR 39 \#3527.

2. N. P. Bhatia and G. P. Szegö, Dynamical systems: Stability theory and applications, Lecture Notes in Math., no. 35, Springer-Verlag, Berlin, 1967, p. 122. MR 36 \#2917.

3. P. Eberlein and B. O'Neill, Visibility manifolds (to appear).

4. L. Green, Geodesic instability, Proc. Amer. Math. Soc. 7 (1956), 438-448. MR 18, 148.

5. - Surfaces without conjugate points, Trans. Amer. Math. Soc. 76 (1954), 529-546.

6. — A theorem of E. Hopf, Michigan Math. J. 5 (1958), 31-34. 
7. G. Hedlund and M. Morse, Manifolds without conjugate points, Trans. Amer. Math. Soc. 51 (1942), 362-386. MR 3, 309.

8. W. Klingenberg, Geodätischer Fluss auf Mannigfaltigkeiten vom hyperbolischen Typ, Preprint, Bonn, Germany, 1970.

9. M. Morse, A fundamental class of geodesics on any closed surface of genus greater than one, Trans. Amer. Math. Soc. 26 (1924), 25-60.

10. —, Instability and transitivity, J. Math. Pures Appl. (9) 14 (1935), 49-71.

Department of Mathematics, University of California, Berkeley, California 94720

Department of Mathematics, University of California, los Angeles, California 90024

Current address: Mathematisches Institut der Universität Bonn, Bonn, Federal Republic of Germany 